\title{
Communications
}

\section{An Umbilical Data-Acquisition System for Measuring Pressures Between the Foot and Shoe}

HONGSHENG ZHU, NABIL MAALEJ, JOHN G. WEBSTER, WILLIS J. TOMPKINS, PAUL BACH-Y-RITA, AND JACQUELINE J. WERTSCH

\begin{abstract}
We have developed an umbilical data-acquisition system for measuring pressures between the foot and shoe during walking. I consists of pressure sensors in the insoles of shoes, amplifier circuits, umbilical cables, an analog-to-digital converter, and a graphics display card in an IBM PC for real-time data collection and display. The applied pressure on a sensor decreases its resistance, which causes the output voltage of the amplifier circuit to increase. We attach seven sensors to the surface of each insole of a pair of extra-depth shoes and calibrate all the sensors in the insole before and after each test using a load cell as a reference. The IBM PC samples the outputs from the sensor and the load cell and stores a piecewise linear lookup table for use in compensation for the nonlinearity of the sensor. On the PC's graphics display, two programs provide displays of foot pressures as real-time bar graphs or as analog pressure versus time curves.
\end{abstract}

\section{INTRODUCTION}

The diabetic foot is especially susceptible to the complications of vascular disease and neuropathy. Patients with diabetic polyneuropathy often lose pain and temperature sensations in their feet. They receive inadequate information about pressures under the feet during walking or standing. Thus, they can injure their feet accidentally without being aware of the injury. Painless trauma devel ops and results in ulceration. Repetitive injury may also produce bone changes, causing the foot to become deformed. With changes in the configuration of the foot, the gait is altered and new pressure points are created, resulting in ulceration at these points. Infection frequently develops in these ulcers, with the risk of progressing to gangrene and amputation [1].

There are two common methods to measure the pressure distribution under the foot during walking. One method uses a force plate system. Usually the force plates are embedded in the floor of a platform. The subject must strike his/her feet within the confines of the measuring surface. Due to the forced walking pattern, the measurements on the force plates are not necessarily representative of the natural gait of the subject. In addition, this system is limited to single-step, barefoot walking. It is impossible to measure pressure for a large number of steps under controlled conditions with this system.

Manuscript received June 13, 1988; revised December 18, 1988. This work was supported by Contract Number V695-P2906 from the Rehabilitation Medicine Service, Veterans Administration Medical Center, Milwaukee, WI 53295.

H. Zhu, N. Maalej, J. G. Webster, and W. J. Tompkins are with the Department of Electrical and Computer Engineering, University of Wisconsin, Madison, WI 53706.

P. Bach-y-Rita is with the Department of Rehabilitation Medicine, University of Wisconsin, Madison, WI 53792.

J. J. Wertsch is with Rehabilitation Medicine Service, Veterans Administration Medical Center, Milwaukee, WI 53295.

IEEE Log Number 9036846.
Another method of measuring the pressure distribution under the foot is to place sensors in the insole of a shoe. This technique can measure the plantar pressures during normal activity of a shoewearing subject. Bauman and Brand taped capacitive pressure pads $100 \mathrm{~mm}^{2}$ in area and $1 \mathrm{~mm}$ thick to the sole of the foot with adhesive tape [2]. These sensors were located beneath the five metatarsal heads, the great toe, and the heel. Hennacy and Gunther built a similar system using piezoelectric sensors in pads $735 \mathrm{~mm}^{2}$ [3]. Lereim and Serek-Hanssen embedded silicon beam strain-gage sensors $12 \mathrm{~mm}$ in diameter and $2.5 \mathrm{~mm}$ in thickness into a PVC insole [4]. Miyazaki and Iwakura put two large rigid rectangular strain gage sensors on the heel region $(65 \times 35 \mathrm{~mm})$ and metatarsal head region $(85 \times 35 \mathrm{~mm}$ ) of each sole of a pair of tennis shoes [5]. There were several problems with the sensor because of its structure, shape, and attachment to the shoe. To solve these problems, they subsequently used a flexible capacitive sensor made of rubber sponge and copper foils [6]. Chizeck et al. embedded four Model 105 strain-gage type miniature pressure sensors into the center of a soft insole supported by a thin and rigid sole to estimate the center of pressure under the foot [7].

Recently, Hermens et al. developed a semiportable system to measure the vertical reaction forces on both feet during walking [8]. They used eight capacitive force sensors attached to the bottom of the sole of the patient's shoes. The force sensors could be easily attached to and removed from the patients' shoes and generally did not influence walking. But this system could only measure several consecutive steps for a period of $20 \mathrm{~s}$. To obtain the pressure distribution over a large area of the sole of the foot, Henning et al. developed a special pressure sensitive insole made of 499 piezoelectric ceramic pressure sensors [9]. Each element of the sensor array was $4.78 \mathrm{~mm}^{2}$ in area and $1.2 \mathrm{~mm}$ thick. They embedded all sensors in a 3-4 $\mathrm{mm}$ thick layer of highly resilient silicone rubber at a center-to-center spacing of $6 \mathrm{~mm}$. This system provided much detailed information about the pressure distribution under the foot.

In an effort to determine the role of pressure in causing damage to insensate diabetic feet, we built an umbilical data-acquisition system to monitor the pressure distribution under the bony prominences of the feet during normal walking in shoes. This system is able to measure the pressures under the feet dynamically for 50 to 60 consecutive steps. It allows the subject to walk freely. The system consists of resistive pressure sensors in the insoles of the shoes, amplifier circuits, umbilical cables, an analog-to-digital converter (ADC), and an IBM PC for real-time data collection and display.

\section{Pressure Sensors and Amplifier Circuits}

We used force-sensitive resistive pressure sensors (Interlink Electronics, 535 E. Montecito St., Santa Barbara, CA 93105), each $16 \mathrm{~mm}$ in diameter, to measure foot pressures [10], [11]. Each sensor consists of a conductive polymer sensing film deposited on Mylar film and an interdigitated conductive pattern printed on an opposing Mylar film. When the conductive polymer is pressed against the conductive pattern, the sensor's resistance decreases. With no applied pressure, the sensor's resistance is in the range of 10-100 M , which decreases with pressure to a minimal value of about $2 \mathrm{k} \Omega$. Each sensor costs less than $\$ 1$ and is only $250 \mu \mathrm{m}$ thick. It is flexible and easily taped on the insoles of the shoes using Scotch tape. In our design, the output voltage of the amplifier circuit increases from 0 to $3.5 \mathrm{~V}$ as the applied pressure causes the sensor's resistance to decrease. The hysteresis of the sensor backed 


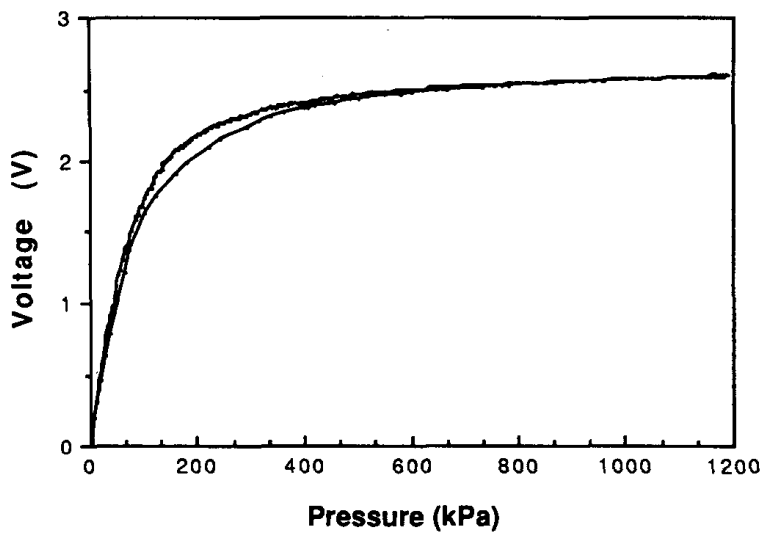

Fig. 1. Increasing sensor pressure yields a nonlinear output voltage.

with metal is $3 \%$ of a $2 \mathrm{MPa}$ full scale. Its sensitivity is $6 \mathrm{mV} / \mathrm{kPa}$ for pressures less than $350 \mathrm{kPa}$ and about $0.15 \mathrm{mV} / \mathrm{kPa}$ for pressures higher than $650 \mathrm{kPa}$.

We taped seven Interlink sensors on the surface of each insole of a pair of P. W. Minor Super X extra-depth shoes. The sensors are located under the center of the heel, the five metatarsal heads, and the big toe of each foot since these bony prominences have higher pressures than other areas.

To locate the bony prominences, we rolled an even layer of stamp pad ink onto a thin ink pad in an Apex foot imprinter (APEX Foot Health Ind., Inc., So. Hackensack, NJ 07606). The subject then walked barefoot on a piece of paper placed on the ink pad. From the footprint on the paper, we located seven high pressure points by choosing the seven relatively darker areas on the paper. Then we attached seven pressure sensors to those points on the insole. We tied a seven-channel amplifier circuit to each of the subject's lower legs using Velcro straps to amplify the signals from the seven sensors under each foot. From the amplifers, two 10-wire shielded flexible cables, each $5 \mathrm{~mm}$ in diameter and $10 \mathrm{~m}$ long, carry the signals to the ADC in the IBM PC while the subject is walking. The cables also supply the operating power to the amplifiers. The cables are anchored at the subject's belt to reduce their effects on the subject's walking. This system can record the foot pressures for $60 \mathrm{~s}$ (about 50 consecutive steps).

We calibrated all the sensors before and after each test using a 440-N load cell as a reference. Fig. 1 shows a calibration curve. Outputs from the sensor and the load cell sampled by the computer generated a piecewise linear lookup table for use in compensation for the nonlinearities of the sensors. We calibrated the sensors in the range of $0-1.5 \mathrm{MPa}$ at a speed of about $3 \mathrm{~s}$ per cycle. Fig. 2 shows the flowchart of the sensor calibration program which is written in the $\mathrm{C}$ language.

Foot Pressure Data acquisition and Display

The Lab Master (Tecmar) signal-acquisition system installed in the IBM PC provides 16 channels of analog-to-digital conversion with 12-b amplitude resolution [12]. The bit resolution is $5 \mathrm{mV}$ providing $2.7-\mathrm{kPa}$ pressure resolution when the peak pressure applied to the sensor is in the range of $500-700 \mathrm{kPa}$. In tests to decide an optimal sample frequency for data acquisition, we used sample frequencies from 10 to $100 \mathrm{~Hz}$ with increments of $10 \mathrm{~Hz}$. We found that peak pressures at $20-\mathrm{Hz}$ and $100-\mathrm{Hz}$ sampling frequencies were within $3 \%$ of each other, so we chose the lower sampling rate of $20 \mathrm{~Hz}$ per channel to maximize the data acquisition time.

Fig. 3 shows the data-acquisition flowchart for the program which is written in the $C$ language. The $\mathrm{PC}$ acquires digital voltage data from the ADC, translates them into meaningful pressures ac-

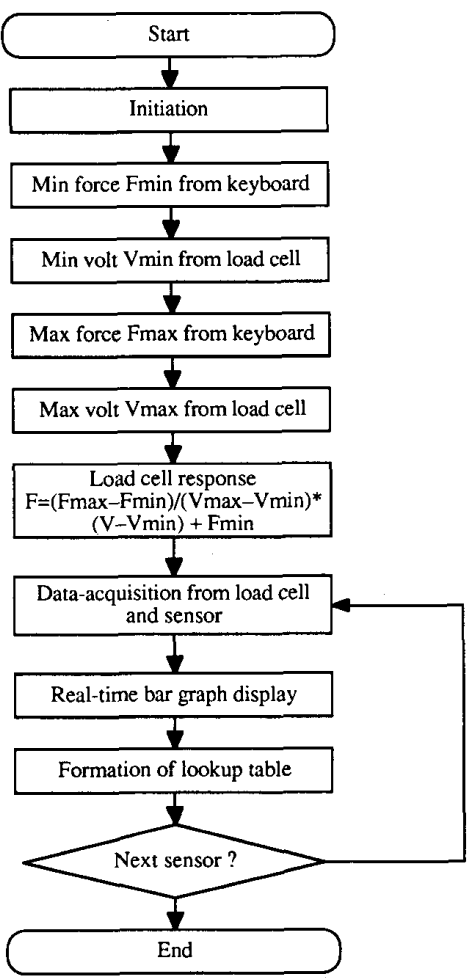

Fig. 2. Sensor calibration flowchart.

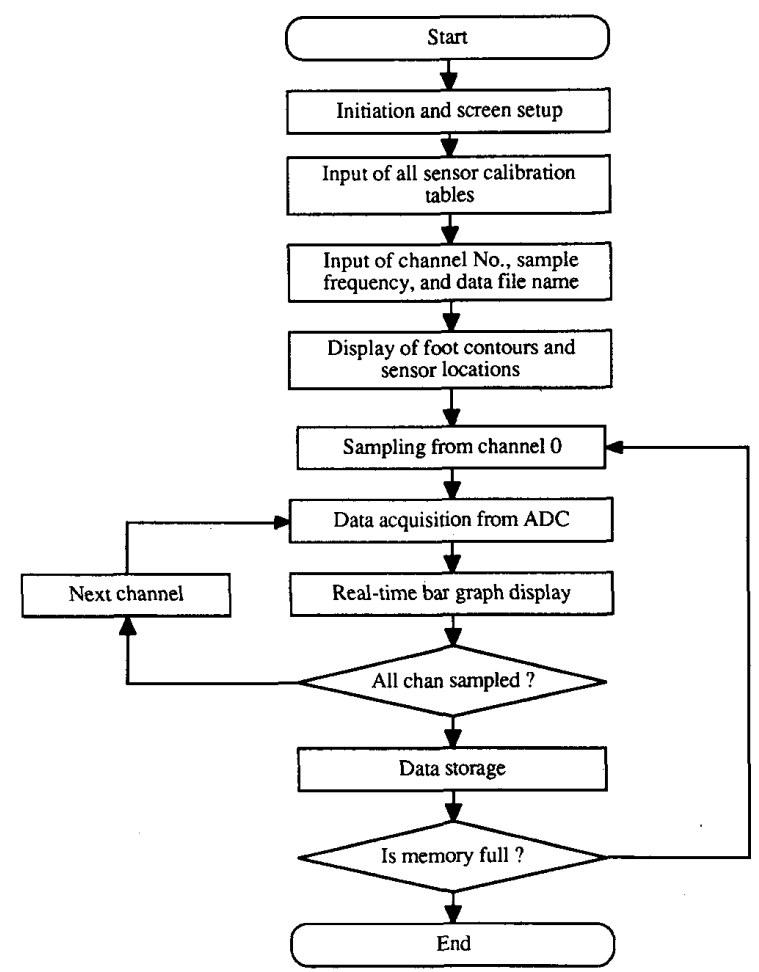

Fig. 3. Data-acquisition flowchart. 


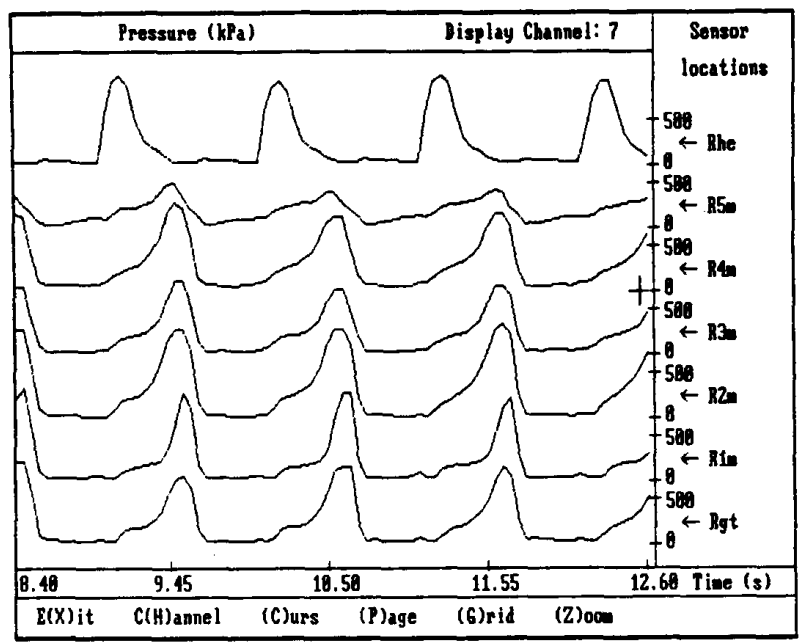

Fig. 4. Pressures under the right foot vary with time.

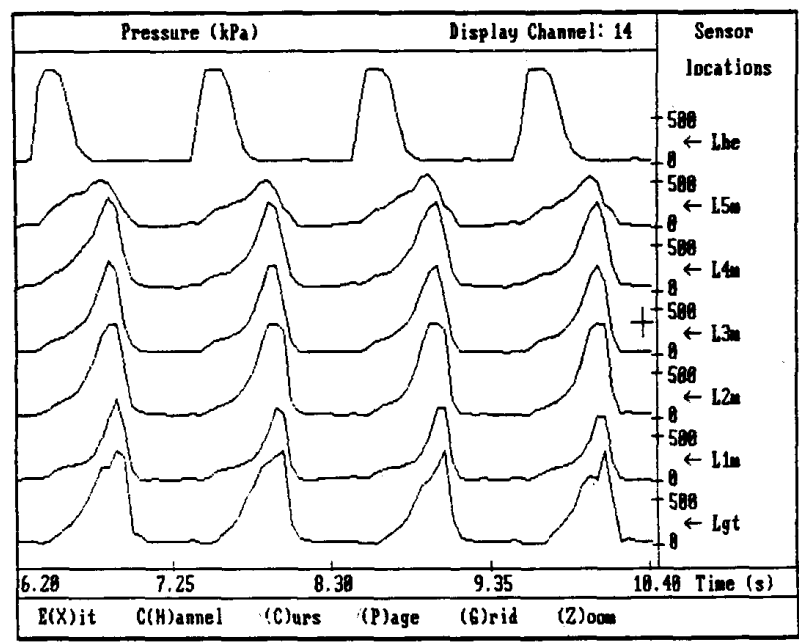

Fig. 5. Pressures under the left foot vary with time.

cording to the prestored sensor calibration cables, and then provides pressure data in real time. The user has the option to select the number of channels to record and the sampling frequency. Sampled data for $60 \mathrm{~s}$ require an 18-kilobyte file for storage.

There are several common methods for presenting foot pressures graphically. One method shows the pressures as bar graphs within a foot outline. Hermens et al. outlined the left and right feet on the computer screen with a bar graph at the location of each sensor [8]. The height of the bar represented the amplitude of force at a given time. The measured forces could be reviewed at 200 -ms time intervals, forward or backward, in real time or in slow motion. The advantage of this real-time display was that it allowed a direct but imprecise view of pressures. A disadvantage was that it was impossible to capture such a dynamic picture on paper. The most commonly used method of presenting data is to plot pressure versus time for all sensors [2], [8], [13], [14]. This shows the temporal relationships of all the pressure waveforms. Another useful method is to display the total force per foot as a function of time $[5],[6]$,
[8]. The total force is calculated from the sum of the individual forces from all sensors under each foot. However, such a total force does not represent the actual total force under each foot since the sensors do not cover the whole area of the foot.

One method of presenting the measured forces is to calculate the center of force of the individual forces of one foot as a function of time [8], [15], [16]. This method gives a visual representation of the measured forces under each foot and shows how the center of the force distribution moves under the foot during the stance phase. Another way of presenting data is 3-D computer graphics of pressure contours under the foot [9], [16], [17]. The intersections of the lines of a grid superimposed on the shoe outline represent the locations of the centers of the sensor elements. An interpolation procedure determines the complete vertical pressure distribution at any instant. The height of the surface above the ground plane is directly proportional to pressure. This method presents data in a visually meaningful manner. However it is very computationally intensive. Franks et al. used seven different colors on a color mon- 


\begin{tabular}{|l|c|c|}
\hline \multicolumn{1}{|c|}{ Locations } & Right foot & Left foot \\
\hline Center of heel & 651 & 633 \\
\hline Fifth metatarsal & 626 & 595 \\
\hline Fourth Metatarsal & 590 & 600 \\
\hline Third metatarsal & 590 & 617 \\
\hline Second metatarsal & 716 & 742 \\
\hline First metatarsal & 720 & 752 \\
\hline Great toe & 630 & 640 \\
\hline
\end{tabular}

Fig. 6. Averaged peak pressures $(\mathrm{kPa})$ for a normal subject.

itor to present different pressure levels [18]. Soames et al. displayed pressure-time area, which reflects both pressure and time information, versus the sensor position [19].

We provide two methods of presenting pressure data with the umbilical system. One displays real-time bar graphs at the locations of each sensor. The other shows pressures versus time under each foot. The real-time display allows us to monitor the pressures while the subject is walking or standing. The pressures appear as seven bars within each foot outline. The amplitude of each bar is proportional to the pressure at the particular sensor.

We also play back recorded pressures versus time. This permits analysis of recorded pressures after the experiment. Figs. 4 and 5 show the pressures of seven sensors under the right foot and left foot respectively when a subject is walking normally. Keyboard options provide for display of any one or combination of the seven channels to study time and pressure relationships. Other options select different pages of data to display, zoom the display, and put grids on the backgrounds.

\section{Test Results and Discussion}

We tested a normal subject to determine peak pressures during walking. The subject walked seven to ten steps at a cadence of about $60 \mathrm{steps} / \mathrm{min}$. Fig. 6 shows the results. Although peak pressures under the feet depend on many factors such as body weight, walking style, and foot contours, the peak pressures that we measured are in the range of $500-1000 \mathrm{kPa}$ and are consistent with those reported by other researchers [16], [19]-[21].

This umbilical system for monitoring pressure distribution under the normal foot has several limitations. First, the subject can only walk within a limited distance and only seven to ten steps before turning because of the length of the umbilical cables. Frequent turns affect the subject's normal gait pattern. Second, the use of cables might also affect the subject's normal gait.

With the advent of the Electrodynogram (EDG), a computerized gait analysis system developed by the Langer Biomechanics Group, Inc., physicians can objectively quantify biomechanically generated pressures of the foot at the interface of the foot and appropriate surfaces and make a diagnosis based on statistical data [22]. The EDG records forces generated through the feet using a waist-pack recorder. The recorder uses a CMOS microprocessor and an 8-kilobyte solid-state memory [23].

We are building a similar battery-powered system with an expanded memory of 480 kbytes, which can continuously sample at a $20-\mathrm{Hz}$ rate per channel and store signals from 16 sensor channels for $15 \mathrm{~min}$. After that the data are transferred to the IBM PC for later analysis and display in about $60 \mathrm{~s}$ through a parallel data link. Such a microprocessor-based data-acquisition system will enable us to monitor the pressure distributions as a function of time inside the shoes on freely-moving ambulatory subjects.

The portability of the system will offer more potential for studying pressure variations under the foot than does the current umbil- ical system. We plan to collect and analyze pressure and timing information under both normal and insensate feet. By comparing the difference of pressures under the feet and timing of footsteps for normal and insensate subjects, we will determine what roles pressure and timing play in tissue damage under the foot. Then we will feed back the most important information to the patients through electrotactile stimulation to help the diabetic protect his/ her feet from tissue damage.

\section{REFERENCES}

[1] M. E. Levin, "Medical evaluation and treatment," The Diabetic Foot, M. E. Levin and L. W. O'Neil, Eds. St. Louis, MO: C. V. Mosby Co., 1983, pp. 1-61.

[2] J. H. Bauman and P. W. Brand, "Measurement of pressure between foot and shoe," Lancet, pp. 629-632, 1963.

[3] R. A. Hennacy and B. S. M. S. Gunther, "A piezoelectric crystal method for measuring static and dynamic pressure distribution in the feet," J. Amer. Podiatry Ass., vol. 65, pp. 444-449, 1975.

[4] M. D. Lereim and F. Serek-Hanssen, "A method of recording pressure distribution under the sole of the foot," Bull. Pros. Res., vol. 20 , pp. 118-125, 1973.

[5] S. Miyazaki and H. Iwakura, "Foot-force measuring device for clinical assessment of pathological gait," Med. Biol. Eng. Comput., vol. 16, pp. 426-436, 1978.

[6] S. Miyazaki and A. Ishida, "Measurement of vertical foot force," Med. Biol. Eng. Comput., vol 22, pp. 309-316, 1984.

[7] H. J. Chizek, P. M. Selwan, and F. L. Merat, "A foot pressure sensor for use in lower extremity neuroprosthetic development," presented at RESA 8th Annu. Conf., 1985, pp. 379-381.

[8] H. J. Hermens, C. A. deWaal, J. Buurke, and G. Zilvold, "A new gait analysis of system for clinical use in rehabilitation center," $O r$ thoped., vol. 9, pp. 1669-1675, 1986.

[9] E. M. Hennig, P. R. Cavanagh, H. T. Albert, and N. H. Macmillan, "A piezoelectric method of measuring the vertical contact stress beneath the human foot," J. Biomed. Eng., vol. 4, pp. 213-222, 1982.

[10] N. Maalej, H. Zhu, J. G. Webster, W. J. Tompkins, J. J. Wertsch, and P. Bach-y-Rita, "Pressure monitoring under insensate feet," in Proc. Annu. Conf. IEEE Eng. Med. Biol. Soc., 1987, pp. 1823-1824.

[11] R. Riegert, "The force sensing resistor: A new tool in sensor technology," in Proc. Sensors Expo, Chicago, IL, 1986, pp. 143-146.

[12] Tecmar Inc., PC-Mate Lab Master: Installation manual users guide. Tecmar Inc., 6225 Cochran Road, Solon, OH 44 139, 1983.

13] A. J. M. Boulton, C. A. Hardisty, R. P. Betts, C. I. Franks, R. C. Worth, J. D. Ward, and T. Duckworth, "Dynamic foot pressure and other studies as diagnostic and management aids in diabetic neuropathy," Diabetics Care, vol. 6, pp. 26-33, 1983.

[14] I. A. F. Stokes, I. B. Fairs, and W. C. Hutton, "The neuropathic ulcer and loads on the foot in diabetic patterns," Acta. Orthop. Scand., vol. 46, pp. 839-847, 1975.

[15] M. G. Blackburn, P. A. Tosh, R. D. McLeish, and L. Smidt, "An investigation of the centers of pressure under the foot while walking," J. Bone Joint Surg., vol. 57-B, pp. 98-103, 1975.

[16] P. R. Cavanagh, and A. Michiyoshi, "A technique for the display of pressure distributions beneath the foot," J. Biomech., vol 13, pp. 69$75,1980$.

[17] H. Gerber, "A system for measuring dynamic pressure distribution under the human foot," J. Biomech., vol. 15, pp. 225-227, 1982.

[18] C. I. Franks, R. P. Betts, and T. Duckworth, "Microprocessor-based image processing system for dynamic foot pressure studies," Med. Biol. Eng. Comput., vol. 21, pp. 566-572, 1983.

[19] R. W. Soames, C. D. Blake, J. R. R. Scott, A. Goodbody, and D. A. Brewerton, "Measurement of pressure under the foot during function," Med. Biol. Eng. Comput., vol. 20, pp. 489-495, 1982.

[20] P. R. Cavanagh, M. M. Rogers, and A. Liboshi, "Pressure distribution under symptom-free feet during barefoot standing," Foot and Ankle, vol. 7, pp. 197-217, 1987.

[21] R. W. Soames, "Foot pressure patterns during gait," J. Biomed. Eng., vol. 7, pp. 120-126, 1985

[22] P. Maffei and M. R. Power-Barnes, "Computerized gait analysis: A quantitative diagnostic tool," Diagnosis, vol. II, pp. 23-27, 1984.

[23] M. Polchaninoff, "Gait analysis using a portable, microprocessorbased segmental foot force measuring system," in Proc. IEEE Seventh Annu. Symp. Comput. Appl. Med. Care, 1983, pp. 897-899. 